\title{
Simplificación de la tarea de picking industrial para el diseño de un sistema robótico.
}

\author{
Majd Kassawat, Angel J. Duran, Enric Cervera \\ Robotic Intelligence Lab, Universitat Jaume I, (España), \\ $\{$ majd, abosch, ecervera $\} @ u j i . e s$
}

\begin{abstract}
Resumen
La industria de picking ha experimentado un gran auge debido al éxito de las compañías de comercio electrónico. El proceso de preparado de pequeños pedidos comporta una complejidad inherente debida a la variabilidad de los productos en cuanto a sus formas, colores, texturas y pesos. En este documento se van a discutir diferentes aspectos de este problema y se va a proponer una solución basada en la combinación de tres elementos: un sistema robótico colaborativo con dos brazos, una mesa de preparación de pedidos y un sistema de almacenaje adaptado. Esta solución se evalúa siguiendo las reglas y restricciones propuestas en el Amazon Robotics Challegence 2017 y se compara con la solución elaborada para el Amazon Picking Challenge 2015, con el objetivo de ilustrar las mejoras de rendimiento cuando el problema se adapta al sistema robótico.
\end{abstract}

Palabras clave: Picking, Sistema robótico, Integración sistemas, Industria 4.0

\section{Introducción}

La creciente demanda por parte de los consumidores de productos provenientes de comercio electrónico, ha provocado que las empresas dedicadas a este sector de negocio requieran de sistemas de preparación de pedidos automatizados. Estos sistemas varían en escala, funcionalidad y eficiencia. Las pequeñas y medianas empresas (PYMES), con un número limitado de productos, pueden separarlos durante el proceso de almacenaje, lo que posibilita que la posterior localización y selección se haga simplemente por su posición en el almacén. No obstante, en el caso de grandes compañías de comercio electrónico con un rango de productos muy amplio,la separación de esos productos en localizaciones específicas dentro de los almacenes, se convierte en una solución inviable. Grandes empresas lideran el desarrollo de tecnologías y sistemas automáticos capaces de preparar cientos de miles de ordenes al día. La compañía Amazon ha automatizado parcialmente sus almacenes mediante flotas de plataformas robóti- cas móviles que permiten transportar estanterías (figura 1) llenas de objetos distribuidos en localizaciones aleatorias, hasta la zona de picking. En este punto, un operario, siguiendo sus órdenes de preparación, identifica, selecciona y coloca los objetos en las correspondientes cajas para ser enviados a los clientes.

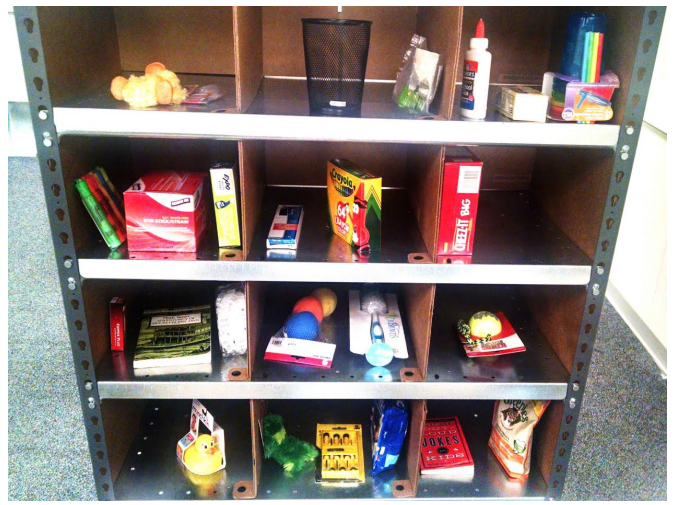

Figura 1: Ejemplo de estantería usada por Amazon en sus almacenes. Este caso particular es una muestra de la estantería usada en el Amazon Picking Challenge de 2015

Durante los últimos tres años, la división de este compañía dedicada a la investigación en robótica (Amazon Robotics), ha propuesto desafíos para realizar tareas de picking mediante sistemas robóticos, en grado de dificultad creciente. Así, en el primer desafío, planteado en el año 2015, Amazon Picking Challenge (APC), suministraba una estantería en la que se colocaban, de forma manual, objetos diferentes en cada contenedor. El robot debía colocar en un caja los objetos detallados en una orden de trabajo específica. Se usó la misma estantería en el desafío de 2016, pero en esta edición, los sistemas robóticos propuestos por los participantes debían realizar una tarea de picking sobre los objetos de una caja, para colocarlos en la estantería (stowing task). En esta segunda edición se produjo un incremento en el número y variedad de objetos a usar por los sistemas presentados. Los equipos participantes afrontaron el desafío con diferentes propuestas: Team Nanyang, MIT y NimbRo usaron un sólo brazo robótico con una gran área de trabajo [3], [14], [16]. Los equipos, Team 
$K$ y Team Duke usaron un manipulador dual comercial denominado Baxter de Rethink Robotics. Otros equipos presentaron sistemas móviles sobre ruedas o raíles que facilitaban la planificación del movimiento del brazo robótico [4],[7]. El factor determinante a la hora de elegir el tipo de sistema para facilitar la planificación de los agarres, fue la capacidad del robot de acceder a todos los contenedores de la estantería desde diferentes ángulos. En la última competición, celebrada en 2017, se modificó de nuevo el desafío y, además de incluir las tareas de picking y stowing, había que diseñar un sistema de almacenaje que pudiera albergar hasta 20 objetos diferentes que debían ser fácilmente transportados por los robots Kiva usados por Amazon en sus almacenes [14].

En general, estos desafíos giran en torno a dos grandes problemas relacionados con la visión y el agarre. El primero de ellos (el reconocimiento de objetos) es un problema clásico [10],[15]. En el segundo problema están implicadas varias disciplinas dentro del campo de la robótica: manipulación complaciente [13], control cinemático de robots bimanipuladores [12], planificación de trayectorias [11], [17] y técnicas de agarre [2].

Finalmente, se ha propuesto el uso de esta competición como un sistema de evaluación y comparativa [8] para resolver un problema específico pero que permite evaluar los avances en robótica dentro de los campos citados anteriormente.

En este artículo se expone el problema planteado por Amazon en el desafío Amazon Robotic Challenge (ARC) del año 2017 (sección 2), así como las soluciones aportadas (sección 3) por nuestro equipo Robinlab UJI en esta competición, que están basadas en la reducción de la complejidad mediante la optimización del sistema de almacenaje y en la cooperación entre dos sistemas robóticos: un robot con dos manipuladores (Baxter) y una plataforma móvil de tres grados de libertad, que hace las funciones de mesa de preparación de pedidos (Rrubert) (figura 3). En las pruebas experimentales se evalúa el rendimiento del sistema, comparándolo con el ganador del ARC 2017. También se realiza una comparativa entre este sistema y el propuesto para el APC 2015 (sección 5). Finalmente se discuten los resultados obtenidos y se obtienen las conclusiones derivadas de las comparativas realizadas (sección 6 ).

\section{Reglas del Amazon Robotic Challenge 2017}

El desafío 2017 consistió en el desarrollo de un sistema robótico que, de manera autónoma, pudiera realizar tres tareas: una tarea de picking, una ta- rea de stowing y una combinación de ambas en una ronda final. Los sistemas robóticos se evaluaron en función del número de objetos recogidos o almacenados de manera correcta para un tiempo dado. Amazon proporcionaba una base de datos de 40 objetos (conjunto de entrenamiento) que presentaban una gran variedad de formas, colores, texturas, pesos y materiales. Se incluyeron objetos difíciles de reconocer por su falta de textura o por impedir la captura de imágenes RGBD debido a su composición (plástico transparente). La selección final para la competición (conjunto de competición) se realizó de forma aleatoria y sólo incluía la mitad de todos los objetos posibles. La otra mitad, desconocida hasta media hora antes de comenzar la competición, estaba compuesta por una serie de objetos similares a los del conjunto de entrenamiento. Este conjunto competición era reconstruido para cada una de las fases del desafío. En este trabajo, para poder comparar los resultados con los del APC 2015, nos vamos a centrar en la parte del picking. Para la tarea de picking, los organizadores escogían 20 objetos del conjunto de competición, que se colocaban manualmente en el sistema de almacenaje, registrándose la ubicación de cada objeto. A continuación, la organización generaba una orden de preparación con 10 objetos, que se tenían que colocar en tres cajas de tamaños diferentes, que sólo se conocían media hora antes del comienzo del desafío. El robot disponía de 15 minutos para empaquetar los objetos de los tres pedidos en cada una de las cajas indicadas en la orden.

\section{Diseño del sistema}

\subsection{Sistema de almacenaje}

En el ARC del 2017, los equipos debían diseñar y construir un sistema propio de almacenaje que, hipotéticamente, se usaría con posterioridad en los almacenes de la compañía. La organización indicaba una serie de restricciones del diseño: i) El volumen de la totalidad del sistema no debía superar los $95.000 \mathrm{~cm}^{3}$. ii) Debía de tener una longitud, anchura y altura inferior a los $125 \mathrm{~cm}$. iii) El número de compartimientos dentro del sistema de almacenaje debía estar comprendido entre 2 y 10 .

A parte de las restricciones físicas marcadas por la organización, se tuvieron en cuenta otros condicionantes derivados del análisis de la especificación de las tareas a desarrollar:

Tareas de visión: La identificación y localización de objetos son algunas de las tareas más complejas y costosas desde el punto de vista del tiempo de procesamiento. Esta complejidad puede ser re- 
ducida evitando al máximo los solapamientos de objetos y las ocultaciones parciales de los mismos. Por ello uno de los criterios de diseño que se tuvo en cuenta a la hora de construir el sistema de almacenaje, fue maximizar la superficie de trabajo, dentro de las limitaciones impuestas por la organización. Este requisito se satisfizo incrementando el número de compartimentos y variando la altura de los mismos en función de la distribución de alturas de los objetos que componían el conjunto de entrenamiento (figura 2).

Tareas de planificación de trayectorias y agarre: Considerar una estantería llena de objetos como la mostrada en la figura 1, implica gran complejidad a la hora de acceder a los objetos más internos. También se presentan problemas de agarre de los objetos, ya que si estos no se sujetan firmemente, se deslizan, pudiendo caer fuera de la estantería. Por este motivo se planteó un diseño para facilitar el agarre vertical, donde una caída del objeto dentro de la zona de trabajo es más fácilmente recuperable. Se diseñó una estantería

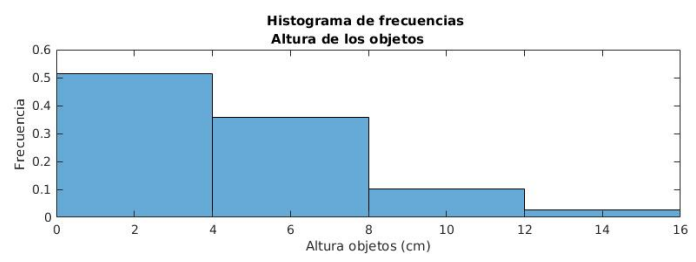

Figura 2: Histograma de frecuencias para las alturas de los objetos que componen el conjunto de entrenamiento

con cajones extraíbles, teniendo en cuenta tanto las limitaciones en las dimensiones impuestas por la organización como la distribución de alturas de los objetos del conjunto de entrenamiento. La altura que definía el volumen disponible dentro de la estantería era de $59 \mathrm{~cm}$. En este volumen se repartieron 5 cajones, de los cuales 3 tenían una altura disponible de $9 \mathrm{~cm}$. entre su base y la base del cajón superior. De esa forma el $80 \%$ de los objetos se podían almacenar en este espacio. Completaban el diseño otro cajón de $14 \mathrm{~cm}$, en el que aproximadamente se podían almacenar el $95 \%$ de los objetos, y un cajón de $16 \mathrm{~cm}$, en el que cabían el $100 \%$ de los objetos. Todos los cajones se fabricaron con plástico transparente para maximizar la visibilidad de los objetos. La utilización del plástico contribuye a la segmentación que realiza el sistema de visión, ya que este material absorbe la radiación infrarroja que proyecta la cámara RGBD, con lo que los cajones son invisibles para la generación de las nubes de puntos.

\subsection{Diseño de la mesa de preparación de pedidos}

La mesa de preparación de pedidos (rRubert), es un sistema robótico que puede ser dividido en dos partes: la zona de almacenaje de cajas y la zona de trabajo. Mientras que la primera sólo tiene un grado de libertad a lo largo del eje X, la segunda tiene 3 grados de libertad que permiten desplazar los cajones desde la estantería a una posición que facilita los agarres y el reconocimiento de objetos (figura 3). La zona de trabajo se desplaza en el eje $\mathrm{Z}$ para alinearse adecuadamente con la altura de cada cajón. Se utilizan 5 sensores de infrarrojos para localizar cada posición de los cajones. Una vez la zona de trabajo está alineada con el cajón que se desea extraer, actúa el eje Y, de tal forma que empuja el cajón para introducirlo en la estantería o lo succiona para extraerlo mediante un sistema de vacío (figura 4).

El eje X coloca el cajón extraído del sistema de almacenaje, en la posición adecuada para la captura de la imagen y de la nube de puntos por parte del sistema de visión. Las cámaras RGBD están colocadas estáticas en la parte superior de la zona de trabajo. Esta posición se estimó mediante la intersección de los volúmenes alcanzables por ambos brazos utilizando la cinemática inversa e intentando mantener la penúltima articulación del Baxter lo más vertical posible. Los movimientos a lo largo de los ejes X e Y se consiguen usando dos motorreductores de 24 voltios. El movimiento en el eje Z, lo lleva a cabo por un motorreductor de engranajes de 24 voltios. Dos encoders asociados a los motores de los ejes X e Y permiten mantener la velocidad de traslación constante, incluso si se produce una variación en el peso de los cajones, debida a la diversidad de los objetos que contienen.

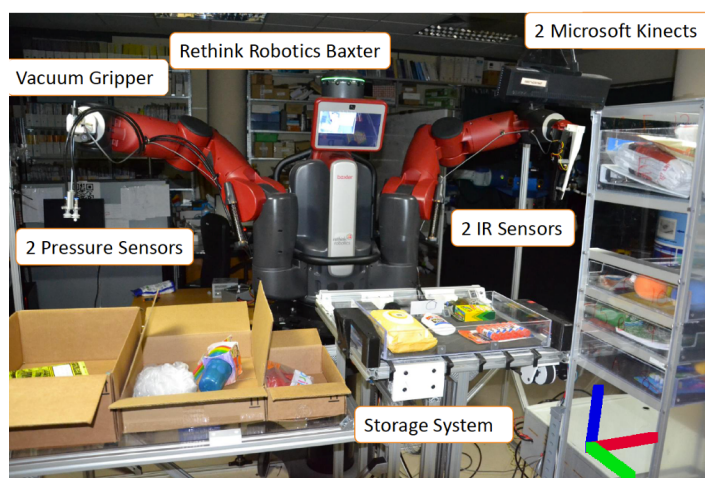

Figura 3: Sistema robótico propuesto por el equipo Robinlab UJI para afrontar el desafío de Amazon Robotic Challenge 2017. Sistema de referencia: rojo (eje X), verde (eje Y) y azul (eje Z). 


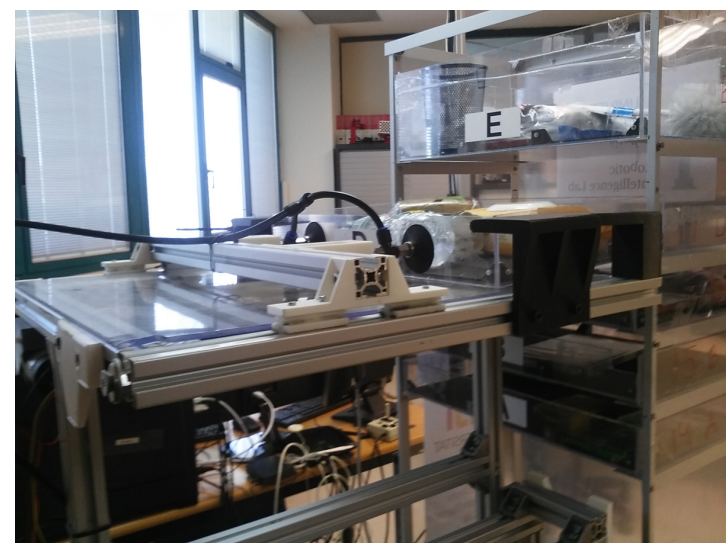

Figura 4: Detalle del sistema de extracción de cajones implementado en la mesa de preparación de pedidos.

\subsection{Diseño de los sistema de agarre del Baxter}

La plataforma que se usa para ejecutar los agarres es un manipulador (Baxter) con dos brazos y 7 grados de libertad cada uno. Unos sensores integrados en cada brazo proporcionan la información de posición y torque de cada articulación. Esta información se usa para detectar y evitar las posibles colisiones, tanto consigo mismo como con elementos del entorno. La carga máxima soportada por cada brazo es de $2.5 \mathrm{~kg}$ en su extremo. Este hecho limita los diseños de los sistemas de agarre. Dado que el objeto más pesado propuesto por la organización era de $2 \mathrm{Kg}$, el sistema compuesto por agarre y sensores no debía superar los $0.5 \mathrm{~kg}$. Teniendo en cuenta estas restricciones se equipó a cada brazo con un tipo diferente de sistema de agarre:

Sistema de succión: Consiste en dos ventosas de succión conectadas de manera independiente a dos bombas de vacío (figura 5). A la salida de cada bomba se conectan dos sensores que miden la caída de presión cuando se produce el vacío de succión y así detectan si el objeto ha sido agarrado. Las dos ventosas independientes y redundantes permiten aumentar la probabilidad de éxito durante la succión de los objetos. El sistema de succión incorpora un pequeño sensor de peso, que permite medir la fuerza sólo en la componente en Z. Esto posibilita la diferenciación de algunos objetos con formas regulares, ya que el peso aproximado del objeto es un dato proporcionado por los organizadores.

Sistema de pinza de apriete: El Baxter viene preparado de fábrica para incorporar un pinza de agarre. Ahora bien, debido a la limitada apertura de la misma, sólo es útil para un número reducido de los objetos propuestos, que por otra parte, son perfectamente agarrados por el sistema de vacío. La idea perseguida con este sistema, era agarrar los objetos que no podían ser succionados. En general, estos objetos son los que ofrecen superficies irregulares o deformables (por ejemplo tela). Por este motivo se diseñó una pinza que pudiera "pellizcar"los objetos y aprovechar la propiedad de deformabilidad que era justamente la que presentaba mayores inconvenientes para el agarre mediante el sistema de vacío. Así, aunque la máxima apertura era de $2 \mathrm{~cm}$, se consiguió que el torque que efectuaba el motor del sistema de agarre de Baxter fuera lo suficientemente alto para asegurar el éxito de la operación. Dos sensores infrarrojos instalados en la pinza detectaban si se había agarrado con éxito el objeto.
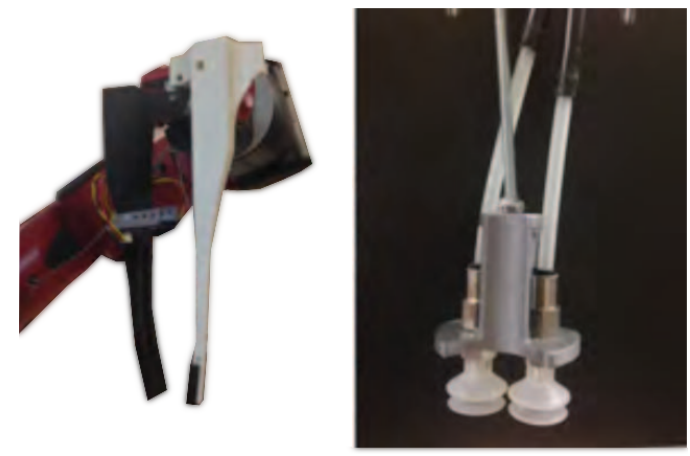

Figura 5: Detalle del sistema de pinza por apriete (izquierda) y del sistema de succión (derecha).

\section{Algoritmos empleados}

Para realizar las tareas de planificación de los agarres y reconocimientos de objetos se usaron una serie de algoritmos. A continuación se exponen brevemente cada uno de ellos.

\subsection{Algoritmos de visión}

\subsubsection{Comparación de características}

La comparación de características usa SIFT (Scale Invariant Feature Transfrom) para identificar los objetos [9]. A partir del procesamiento de las imágenes de la vista superior e inferior de los objetos proporcionadas por Amazon se extraen las características más relevantes y se identifican mediante SIFT. Cuando se busca un determinado objeto dentro de uno de los cajones, se exploran las características particulares de ese objeto para identificarlo. La principal ventaja de este método es que permite determinar la posición y orientación del objeto, aunque esté parcialmente ocluido. $\mathrm{Su}$ principal desventaja reside en que no es aplicable a los objetos sin características destacables (por ejemplo objetos sin textura). 


\subsubsection{Red neuronal profunda SegNet}

Para superar el inconveniente de la detección de objetos sin textura, se recurrió al uso de técnicas de aprendizaje profundo. Así la red SegNet (Segmentation Based Convulotional Networks) [1], permite la segmentación de objetos en una imagen. Se entrenó una red SegNet por cada objeto. En el conjunto de entrenamiento estaba el objeto problema, mezclado con el resto, pero sólo se etiquetaba el objeto correspondiente a la red. El resto se identificaba como fondo. Este tipo de red, en comparación con la otra red neuronal utilizada, es bastante rápido de entrenar, aunque produce falsos positivos para áreas similares de pequeños objetos.

\subsubsection{Red neuronal ResNet}

ResNet (Residual network), es una red neuronal profunda [6], que devuelve la probabilidad de que un pixel de la imagen sea de una determinada clase de objeto. En principio, la red trabaja con las 40 clases de objetos conocidos. Se modificó la última capa de información de los objetos, incluyendo la información proporcionada sobre los objetos presentes en cada cajón para condicionar su probabilidad. De esa forma, si el objeto no estaba, la probabilidad de que un pixel fuera de esa clase era nula. El rendimiento de esta red estaba limitado por el número de imágenes necesarias y los largos tiempos de entrenamiento.

\subsection{Algoritmos de planificación del agarre}

Una vez identificados los objetos, el objetivo del desafío, era agarrarlos y depositarlos en diferentes localizaciones. En este caso, se utilizó la información proveniente de la nube de puntos suministrada por el sistema de visión y segmentada a través de las imágenes RGB. A partir de esta nube de puntos, se generó una serie de vectores de aproximación para colocar el efector final de cada brazo en la posición adecuada para ejecutar el agarre.

\subsubsection{Height Accumulated Features}

Se seleccionó el algoritmo Height Accumulated Features (HAF) [5] para la planificación de los agarres de la pinza. HAF se basa en la extracción y clasificación de características de una nube de puntos. Se considera una malla de un centímetro cuadrado sobre los valores de altura máximos proporcionados por la nube de puntos. Se utiliza una máquina de vector soporte previamente entrenada para seleccionar las mejores características para el agarre. Se produce una acumulación de regiones vecinas con similares características para op- timizar los tiempos de cálculo. HAF es adecuado cuando se usa en agarres en los que la componente vertical es importante. Este es justo el caso de la pinza que diseñamos para ejecutar los agarres de apriete.

\subsubsection{Centroid Normals Approach}

Este algoritmo se utilizó principalmente para determinar la planificación del agarre por el sistema de succión. Este tipo de agarre necesita objetos con superficies planas, poco deformables y limpias. No funciona correctamente en superficies porosas, con agujeros o en telas. Para poder ser agarrados mediante dispositivos que empleen este sistema, los objetos deben presentar superficies planas o con radios de curvatura pequeños. Por todo esto, fue necesario el desarrollo de un algoritmo de planificación para este tipo de agarre : Centroid Normals Approach (CNA). La nube de puntos que genera el sistema de visión se agrupa en un grid de voxels. A continuación, dependiendo de la forma predominante del objeto, se aproxima a un cilindro o a un plano. Utilizando estas figuras se combina la información de centroide con la información de la normal en la superficie aproximada para calcular el vector de agarre.

\section{Pruebas experimentales}

Para la realización de las pruebas experimentales, se consideró sólo la tarea de picking, debido a que esta es la única tarea que se contemplaba en el APC 2015. Las pruebas experimentales realizadas se dividen en dos comparativas: determinación de la eficiencia del sistema diseñado, en comparación con el equipo ganador del año 2017 y la comparativa con el sistema diseñado y presentado en 2015.

\subsection{Rendimiento del sistema propuesto para solventar el Amazon Picking Challenge 2017}

Para realizar estas pruebas se prepararon 8 conjuntos de objetos diferentes, procurando que estuvieran representadas todas las tipologías de objetos propuestas por Amazon. A cada objeto del conjunto de entrenamiento de Amazon se le asignó, al menos, un objeto alternativo. Por ejemplo, si el objeto de Amazon era un libro, el objeto alternativo era otro libro, de tamaño similar pero de colores y texturas diferentes. Los 20 objetos definitivos estaban formados por 10 objetos del conjunto de entrenamiento de Amazon y 10 del conjunto de objetos alternativos. La información sobre los objetos concretos que componían el conjunto, sólo estaba disponible 30 minutos antes de comenzar el test. La información proporciona- 
da por Amazon para cada objeto del conjunto de entrenamiento estaba compuesta por: 6 imágenes de alta resolución sobre fondo negro, una nube de puntos con asignación de color, las especificaciones físicas de cada uno (medidas, peso) y la clase a la que pertenecía. Para cada objeto alternativo se preparó exactamente la misma información.

Se procedió a ejecutar los 8 test con el sistema, siguiendo las normas de puntuación aplicadas por Amazon. Los resultados de estos ensayos se pueden ver en la tabla 1. En dicha tabla se indica el porcentaje de objetos conocidos y desconocidos procesados con éxito, así como el tiempo total utilizado por el sistema propuesto para dar por concluida la tarea. En la prueba real de Amazon, este tiempo sólo se tenía en consideración si se había completado correctamente toda la orden de pedido. Ahora bien, el sistema no fue capaz de completar en ningún caso los 10 objetos, pero estaba habilitado para discernir si se iba a completar la tarea o no. Por este motivo, no se agotaron los 900 segundo de tiempo de que se disponía para la realización del desafío. Partiendo de esta premisa, se computó este tiempo para poder compararlo con los resultados del ARC 2017, ya que el equipo ganador terminó antes de tiempo, completando todos los objetos, por lo que obtuvo una puntuación de 257. Este es el valor de referencia utilizado para calcular el rendimiento de nuestro sistema.

Tabla 1: Resultados obtenidos para los 8 test de objetos propuesto siguiendo las reglas del Amazon Robotic Challenge 2017.

\begin{tabular}{c|c|c|c|c|c|c|c|c}
\hline Test & $\begin{array}{c}\text { Objetos } \\
\text { correctos }\end{array}$ & $\begin{array}{c}\text { Objetos } \\
\text { conocidos }\end{array}$ & $\begin{array}{c}\text { Objetos } \\
\text { descono- } \\
\text { cidos }\end{array}$ & $\begin{array}{c}\text { Tiempo } \\
(\mathrm{s})\end{array}$ & $\begin{array}{c}\text { Puntos } \\
\text { por } \\
\text { tiempo }\end{array}$ & $\begin{array}{c}\text { Puntos } \\
\text { Amazon }\end{array}$ & $\begin{array}{c}\text { Estima- } \\
\text { ción } \\
\text { Total }\end{array}$ & $\begin{array}{c}\text { Rendi- } \\
\text { miento }\end{array}$ \\
\hline 1 & $9 / 10$ & $100,00 \%$ & $83,00 \%$ & 536 & 73 & 135 & 207,8 & $80,86 \%$ \\
\hline 2 & $7 / 10$ & $66,00 \%$ & $75,00 \%$ & 869 & 6 & 80 & 86,2 & $33,54 \%$ \\
\hline 3 & $8 / 10$ & $66,00 \%$ & $100,00 \%$ & 520 & 76 & 105 & 181 & $70,43 \%$ \\
\hline 4 & $8 / 10$ & $75,00 \%$ & $83,00 \%$ & 507 & 79 & 95 & 173,6 & $67,55 \%$ \\
\hline 5 & $8 / 10$ & $83,00 \%$ & $75,00 \%$ & 678 & 44 & 90 & 134,4 & $52,30 \%$ \\
\hline 6 & $7 / 10$ & $50,00 \%$ & $100,00 \%$ & 670 & 46 & 110 & 156 & $60,70 \%$ \\
\hline 7 & $9 / 10$ & $100,00 \%$ & $75,00 \%$ & 615 & 57 & 100 & 157 & $61,09 \%$ \\
\hline 8 & $6 / 10$ & $60,00 \%$ & $60,00 \%$ & 881 & 4 & 75 & 78,8 & $30,66 \%$ \\
\hline Media & $7.75 / 10$ & $75,00 \%$ & $81,38 \%$ & 659,5 & 48,1 & 98,75 & 146,85 & $57,14 \%$ \\
\hline
\end{tabular}

\subsection{Comparativa con el sistema propuesto para el Amazon Picking Challenge 2015}

El sistema propuesto para el APC 2015, consistía en un robot Baxter con dos pinzas, que permitían el agarre de los objetos (figura 7. La estantería industrial utilizada por Amazon en sus almacenes (figura 1) estaba fija en frente del robot. Los objetos se colocaban en los diferentes receptáculos procurando que no se solaparan u ocluyesen. En estas condiciones, el robot utilizaba un sensor Kinect anexado a su brazo para realizar un reconocimiento previo de todos los objetos de la estantería para proceder, a continuación a agarrar el objeto que indicaba la orden de proceso y depositarlo en una única caja. A diferencia del ARC 2017, todos los objetos se conocían previamente. Si bien es cierto que en 2015 no se utilizaron los algoritmos de aprendizaje profundo para la identificación y localización de los objetos, sí se usaban algoritmos alternativos basados en la segmentación de nubes de puntos (los objetos no estaban en contacto entre sí) y en la extracción de características, que tenían una tasa de aciertos similar al complejo caso del sistema actual con objectos en contacto o parcialmente ocluidos. Las reglas del APC 2015

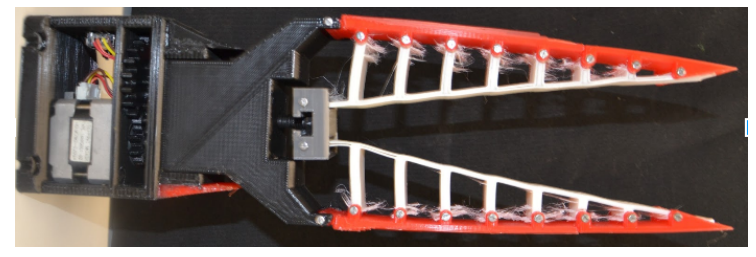

Figura 6: Detalle de la pinza usado en el APC 2015

especificaban que de los 24 objetos presentes en la estantería, se debían colocar en la caja de pedido los 12 seleccionados de una orden de trabajo previa, en un tiempo máximo de 20 minutos. Se prepararon 5 test en los que se escogían de manera aleatoria los 12 objetos a preparar, generando un fichero con las ordenes de trabajo. Se ejecutaron estas pruebas con el sistema del 2015 y con el sistema del 2017. La reglas de puntuación aplicadas fueron las de 2015. Los resultados obtenidos para las cinco ordenes de preparación se pueden ver en la tabla 2 .

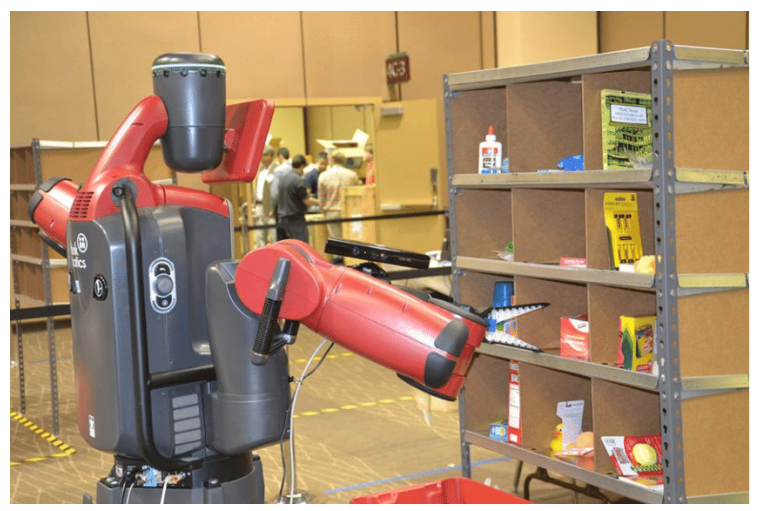

Figura 7: Sistema propuesto para el APC 2015

\section{Discusión y conclusiones}

De los resultados de la tabla 2 se deriva que el rendimiento del sistema propuesto para el APC 2017 es aproximadamente el doble, en todos los aspectos evaluados. Los principales problemas que se presentan en la disposición del sistema del 2015 son la necesidad de planificaciones complejas para 
Tabla 2: Comparativa del sistema propuesto para el APC 2015 con el sistema diseñado para el ARC 2017 utilizando las reglas de puntuación y preparación especificadas en el 2015

\begin{tabular}{ccccc}
\hline \multicolumn{5}{c}{2015} \\
Test & $\begin{array}{c}\text { Objetos } \\
\text { recogidos }\end{array}$ & $\begin{array}{c}\text { Objetos } \\
\text { Reconocidos }\end{array}$ & $\begin{array}{c}\text { Tiempo } \\
\text { empleado (s) }\end{array}$ & $\begin{array}{c}\text { Puntos } \\
\text { Amazon }\end{array}$ \\
\hline 1 & $5 / 12$ & 9 & 1200 & 66 \\
\hline 2 & $4 / 12$ & 8 & 1200 & 40 \\
\hline 3 & $3 / 12$ & 6 & 1200 & 35 \\
\hline 4 & $3 / 12$ & 7 & 1200 & 43 \\
\hline 5 & $4 / 12$ & 7 & 1200 & 38 \\
\hline Media & $3,8 / 12$ & 7,4 & 1200 & 44,4 \\
\hline & & & & Puntos \\
\hline Test & Objetos & Objetos & Tiempo & Amazon \\
\hline 1 & $9 / 12$ & 10 & 530 & 176 \\
\hline 2 & $11 / 12$ & 12 & 670 & 213 \\
\hline 3 & $10 / 12$ & 12 & 600 & 183 \\
\hline 4 & $9 / 12$ & 11 & 520 & 173 \\
\hline 5 & $12 / 12$ & 12 & 750 & 236 \\
\hline Media & $10.2 / 12$ & 11,4 & 614 & 196,2 \\
\hline & & & & \\
\hline
\end{tabular}

acceder a los diferentes cubículos que componen las estantería. Los planificadores de movimientos de los brazos deben tener en cuenta la posibilidad de múltiples colisiones con la estantería, los objetos y el propio robot. Además, una vez alcanzado el punto de aproximación para la ejecución de agarre, el ajuste de la pinza al tamaño del objeto se debe realizar de manera precisa, con el objetivo de no tirar, desplazar o coger otros objetos del mismo receptáculo. En la tabla 2, se puede apreciar que el tiempo de desarrollo de tarea para el sistema de 2015 alcanza el límite de los 20 minutos, sin lograr el número máximo de objetos. El $70 \%$ del tiempo empleado para coger un objeto, se usa en la planificación y ejecución del movimiento, el $10 \%$ se emplea en los algoritmos de visión, y por último, el $20 \%$ restante, en abrir y cerrar la pinza. El tiempo total del ciclo de reconocimiento, agarre y deposito es de 120 s por objeto. Además, debido a la complejidad de las planificaciones y agarres, muchas veces se produce un fallo en esta etapa, con la consiguiente pérdida de todo el tiempo empleado hasta llegar a este punto.

El sistema propuesto para el ARC 2017, permite el diseño de la estantería y, por lo tanto, facilita la adecuación de las especificaciones a la simplificación de los problemas planteados desde el punto de vista del sistema robótico. La utilización de un robot auxiliar (rRubert) con una tarea específica, que consiste en preparar los objetos para facilitar el reconocimiento y agarre de los mismos, se ha demostrado decisiva, tal y como aparece reflejado en la tabla 2. El reconocimiento y localización de un objeto, en este caso necesita apenas un $5 \%$ del tiempo. La extracción del cajón de la estantería, el agarre del objeto y su depósito en la caja suponen el $95 \%$ restante. Un ciclo completo tiene un coste temporal de 37.6 segundos de media por objeto.

En definitiva, se ha realizado la comparativa de dos propuestas de sistema para resolver el problema de la tarea de picking en la preparación de pedidos. El primer sistema (2015) pretendía adaptar el robot al problema, de tal forma que el entorno del robot no esté adecuado a las posibilidades que ofrece el sistema robótico. Por otro lado, la propuesta del 2017, aunque mucho más compleja, resuelve el problema de una manera mucho más eficiente.

Para realizar estas comparativas se han utilizado como base las reglas de los diferentes concursos propuestas por Amazon, así como los objetos y datos proporcionados, lo cual lo convierte en un procedimiento estándar de realizar comparativas en robots que ejecuten este tipo de tareas.

\section{Agradecimientos}

Este documento describe la investigación realizada en el Laboratorio de Robótica Inteligente de la Universitat Jaume I. Este trabajo es financiado por el Ministerio de Economía y Competitividad (DPI2015-69041-R), por el Fondo Europeo de Desarrollo Regional (FEDER) y por la Universidat Jaume I (P1-1B2014-52, PREDOC/2013/06).

\section{English summary}

\section{Simplifying Robotic Picking Tasks in Warehouses by Using a Conveyor- like System}

\begin{abstract}
Item storage and retrieval is forming a crucial part of nearly every industry. The complexity of this process increases dramatically when the stored items differ in shape, color, texture and weight. In this paper we are going to discuss the different aspects of the problem and propose a solution based on a Dual-Arm robotic system, a conveyorlike system and a customized storage system. The items to be picked by the robot have different physical properties that were taken into account while designing the storage system, the conveyor and the grippers.
\end{abstract}


Keywords: Picking task, System Intergration, Industry 4.0

\section{Referencias}

[1] V. Badrinarayanan, A. Kendall, and R. Cipolla. Segnet: A deep convolutional encoderdecoder architecture for image segmentation. IEEE transactions on pattern analysis and machine intelligence, 39(12):2481-2495, 2017.

[2] J. Bohg, A. Morales, T. Asfour, and D. Kragic. Data-driven grasp synthesis - a survey. IEEE Transactions on Robotics, 30(2):289309, 2014.

[3] N. Correll, K. E. Bekris, D. Berenson, O. Brock, A. Causo, K. Hauser, K. Okada, A. Rodriguez, J. M. Romano, and P. R. Wurman. Analysis and observations from the first amazon picking challenge. IEEE Transactions on Automation Science and Engineering, 2016.

[4] N. Correll, K. E. Bekris, D. Berenson, O. Brock, A. Causo, K. Hauser, K. Okada, A. Rodriguez, J. M. Romano, and P. R. Wurman. Lessons from the amazon picking challenge. arXiv preprint arXiv:1601.05484, 2016 .

[5] D. Fischinger and M. Vincze. Empty the basket - a shape based learning approach for grasping piles of unknown objects. In 2012 IEEE/RSJ International Conference on Intelligent Robots and Systems, pages 2051-2057, Oct 2012.

[6] K. He, X. Zhang, S. Ren, and J. Sun. Deep residual learning for image recognition. In Proceedings of the IEEE conference on computer vision and pattern recognition, pages 770 $778,2016$.

[7] C. Hernandez and et al. Team delft's robot winner of the amazon picking challenge 2016 . CoRR, abs/1610.05514, 2016.

[8] J. Leitner, A. W. Tow, N. Sünderhauf, J. E. Dean, J. W. Durham, M. Cooper, M. Eich, C. Lehnert, R. Mangels, C. McCool, et al. The acrv picking benchmark: A robotic shelf picking benchmark to foster reproducible research. In Robotics and Automation (ICRA), 2017 IEEE International Conference on, pages 4705-4712. IEEE, 2017.
[9] D. G. Lowe. Distinctive image features from scale-invariant keypoints. International Journal of Computer Vision, 60(2):91-110, Nov 2004.

[10] E. Martinez-Martin and A. P. del Pobil. Object detection and recognition for assistive robots: Experimentation and implementation. IEEE Robotics Automation Magazine, 24(3):123-138, Sept 2017.

[11] C. Mineo, S. G. Pierce, P. I. Nicholson, and I. Cooper. Robotic path planning for nondestructive testing - a custom matlab toolbox approach. Robotics and Computer-Integrated Manufacturing, 37:1 - 12, 2016.

[12] R. M. Murray. A mathematical introduction to robotic manipulation. CRC press, 2017.

[13] M. Prats. Robot Physical Interaction through the combination of Vision, Tactile and Force Feedback. PhD thesis, $\mathrm{PhD}$ thesis, Universitat Jaume I, 2009.

[14] M. Schwarz, A. Milan, C. Lenz, A. Munoz, A. S. Periyasamy, M. Schreiber, S. Schüller, and S. Behnke. Nimbro picking: Versatile part handling for warehouse automation. In Robotics and Automation (ICRA), 2017 IEEE International Conference on, pages 3032-3039. IEEE, 2017.

[15] M. Schwarz, A. Milan, A. S. Periyasamy, and S. Behnke. Rgb-d object detection and semantic segmentation for autonomous manipulation in clutter. The International Journal of Robotics Research, page 0278364917713117, 2016.

[16] K.-T. Yu, N. Fazeli, N. Chavan-Dafle, O. Taylor, E. Donlon, G. D. Lankenau, and A. Rodriguez. A summary of team mit's approach to the amazon picking challenge 2015. arXiv preprint arXiv:1604.03639, 2016.

[17] A. Zeng, K.-T. Yu, S. Song, D. Suo, E. Walker, A. Rodriguez, and J. Xiao. Multiview self-supervised deep learning for $6 \mathrm{~d}$ pose estimation in the amazon picking challenge. In Robotics and Automation (ICRA), 2017 IEEE International Conference on, pages 1386-1383. IEEE, 2017.

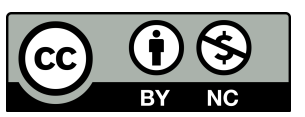

(c) 2018 by the authors. Submitted for possible open access publication under the terms and conditions of the Creative Commons Attribution CC-BY-NC 3.0 license (http://creativecommons.org/licenses/by-nc/3.0/). 